\title{
L-Ferritin targets breast cancer stem cells and delivers therapeutic and imaging agents
}

\author{
Laura Conti ${ }^{1, *}$, Stefania Lanzardo ${ }^{1, *}$, Roberto Ruiu ${ }^{1}$, Marta Cadenazzi ${ }^{1}$, Federica \\ Cavallo ${ }^{1}$, Silvio Aime ${ }^{1}$, Simonetta Geninatti Crich ${ }^{1}$ \\ ${ }^{1}$ Department of Molecular Biotechnology and Health Sciences, Molecular Biotechnology Center, University of Turin, Turin, \\ Italy \\ *These authors have contributed equally to this work \\ Correspondence to: Stefania Lanzardo, email: stefania.lanzardo@unito.it \\ Simonetta Geninatti Crich, email: simonetta.geninatti@unito.it \\ Keywords: ferritin, cancer stem cells, theranostic agents, magnetic resonance imaging, mammary tumors \\ Received: February 23, $2016 \quad$ Accepted: July 18, $2016 \quad$ Published: July 29, 2016
}

\section{ABSTRACT}

A growing body of evidence suggests that cancer stem cells (CSC) have the unique biological properties necessary for tumor maintenance and spreading, and function as a reservoir for the relapse and metastatic evolution of the disease by virtue of their resistance to radio- and chemo-therapies. Thus, the efficacy of a therapeutic approach relies on its ability to effectively target and deplete CSC. In this study, we show that CSC-enriched tumorspheres from breast cancer cell lines display an increased L-Ferritin uptake capability compared to their monolayer counterparts as a consequence of the upregulation of the L-Ferritin receptor SCARA5. L-Ferritin internalization was exploited for the simultaneous delivery of Curcumin, a natural therapeutic molecule endowed with antineoplastic action, and the MRI contrast agent Gd-HPD03A, both entrapped in the L-Ferritin cavity. This theranostic system was able to impair viability and self-renewal of tumorspheres in vitro and to induce the regression of established tumors in mice. In conclusion, here we show that Curcuminloaded L-Ferritin has a strong therapeutic potential due to the specific targeting of CSC and the improved Curcumin bioavailability, opening up the possibility of its use in a clinical setting.

\section{INTRODUCTION}

The occurrence of resistance to chemotherapeutic drugs, tumor recurrences and metastases formation show the difficulties in finding effective cancer treatments and raise the question whether current anti-cancer therapies target the right cancer cell population. There is a growing concern that the commonly used treatments might indeed miss a small population of tumor cells called "tumor-initiating cells" or "cancer stem cells" (CSC), composed of stem-like cells that play a critical role in cancer progression. Like normal stem cells, CSC have the capacity to self-renew and to give rise to a more differentiated progeny, and share common signalling pathways. According to the CSC theory, CSC can be: i) the source of all the tumor cells present in a malignant tumor and ii) responsible for tumor relapse and dissemination, being associated to the resistance to radiotherapy and to the conventional chemotherapeutic agents [1]. CSC have been identified in several human solid tumors and prospectively isolated through specific markers [2], although there is no general consensus on the best markers to identify these cells [3]. In human breast carcinoma, CSC have been identified for the first time by $\mathrm{Al}$ Hajj et al. [4] as a rare population of CD $44^{+} / \mathrm{CD} 24^{-/ \text {low }}$ cells. Furthermore, CSC have the ability to survive and proliferate in anchorage-independent conditions giving rise to non-adherent spheres called tumorspheres that can be selectively cultured and expanded. This property was first described for neuronal progenitors [5] and then extended to progenitor cells of the mammary gland [6], to breast cell lines [7-9], and to human and murine breast carcinomas [10]. Given the central role of CSC in tumor progression, spreading and relapse, the cure for cancer might rely on CSC eradication. On the light of this therapeutic implication, several CSC-targeted approaches 
are being studied. These approaches range from indirect strategies, such as antiangiogenic therapies [1], to direct targeting, pursued through differentiation therapies, reversal of resistance mechanisms [1], and immunotherapy [11-13]. Another promising strategy consists in the identification of new and more specific biomarkers related to CSC status, which could serve as new targets, as we previously described $[8,9]$. Moreover, these targets could be used for the development of new diagnostic tools able to provide an insight into the frequency of CSC within the tumor. Based on evidences obtained from both epidemiological and molecular studies, new insights are emerging linking the presence of excess iron and altered iron metabolism to cancer [14, 15]. Recently, Schonberg et al. [16] have demonstrated an enhanced iron scavenging ability in CSC of glioblastoma multiforme, due to a significant overexpression of the transferrin receptor (TfR) and a consequent increase in transferrin uptake, which is indicative of increased tumorigenicity. Therefore, targeting iron regulation within tumor-specific pathways could represent a potential approach for the development of new effective anti-cancer treatments.

In this study, we exploited the sphere-forming ability of CSC to selectively enrich the stem-like cell population present in a human (MDA-MB-231) and murine (TUBO) breast cancer cell lines, as described in [8], and for the first time we demonstrated that the uptake of L-Ferritin increased in CSC-enriched tumorspheres generated from both cell lines compared to their more differentiated counterparts. We surmise that this behaviour may be associated to their enhanced expression of the L-Ferritin receptor Scavenger Receptor Class A member 5 (SCARA5) that mediates Ferritin endocytosis [17]. Ferritin is the main iron storage protein and is composed of 24 subunits of heavy (H)- or light (L)-chain polypeptides that are present at different ratios in various organs to form a cage architecture of $12 \mathrm{~nm}$ in external diameter, with an inner cavity of $8 \mathrm{~nm}$ [18]. Once deprived of iron, this cavity can be used for the selective delivery of imaging and therapeutic agents to cells expressing Ferritin receptors [19]. The use of these nanotheranostic agents permits the non-invasive analysis of the pharmacokinetics and biodistribution of the nanomedicine formulation. Consequently, it is possible to monitor the efficacy of the therapy in real time and thereby to adapt the treatment regimens [20-22]. Several papers have explored the use of H-Ferritin to deliver selectively doxorubicin to cancer cells $[23,24]$. In this contest, we have previously reported that the selective uptake of native horse spleen Ferritin and Apoferritin (composed by $85 \%$ and $15 \% \mathrm{~L}$ and $\mathrm{H}$ chains, respectively) loaded with MRI contrast agents and the anticancer drug Curcumin in the human breast cancer cell line MCF7 causes a significant reduction in cell proliferation in vitro [25]. Curcumin has been selected as therapeutic agent since it has been reported to exhibit anticancer activity in vitro and to be highly tolerated when administered to patients [26]. However, its poor water solubility and low bioavailability hampers its use as anti-cancer drug [27]. Therefore, loading Curcumin into Apoferritin can represent a solution for its delivery to cancer cells in vivo. Herein, we show that Apoferritin can be exploited for the simultaneous delivery of Gdbased MRI contrast agents and Curcumin for breast CSC targeting. Moreover, we show that administration of Curcumin-loaded Apoferritin leads to the regression of breast tumors in vivo. This approach could potentially enhance the responsiveness to current anticancer treatment regimens and might reduce the risk of relapse and dissemination of the disease.

\section{RESULTS AND DISCUSSION}

\section{SCARA5 is upregulated in breast CSC}

A transcriptional analysis comparing the transcription profile of Her $2^{+}$murine TUBO cells cultured as monolayer with those of the first three in vitro passages of their derived CSC-enriched tumorspheres using MouseWG-6 v2.0 Illumina beadchips (GSE21451) [28] proved that SCARA5 is upregulated in tumorspheres (Figure 1A). SCARA5 protein expression increased from TUBO to tumorsphere-derived cells, as demonstrated by the representative images (panel $\mathrm{B}, \mathrm{C}$ ) and by the quantification of fluorescence intensity (panel D) reported in Figure 1. This enhanced expression is not restricted to TUBO-derived CSC, as it was also observed in tumorspheres derived from human triple negative breast cancer (TNBC) cell line MDA-MB-231 (Figure 1E-1G), further suggesting that SCARA5 may be a promising target of breast CSC.

\section{Breast CSC internalize more Ferritin than differentiated cancer cells}

Since SCARA5 mediates L-Ferritin uptake [29], the first step of this study was the evaluation of the ability of MDA-MB-231 and TUBO tumorspheres to take up Ferritin from the medium compared to their corresponding more differentiated cells. For this purpose, horse spleen Ferritin, composed mostly of L-Ferritin chains and containing ca. 1000 iron atoms per protein, was used without any further modification. The experimental protocol consisted in the measurement of the amount of iron internalized by cells upon 24 hours of incubation in Ferritin-containing medium. The amount of internalized Ferritin was assessed by the ICP-MS determination of the intracellular iron content. The amount of internalized iron was significantly higher in tumorspheres than in differentiated cells, and increased with Ferritin concentration in both TUBO (Figure 2A) and MDA-MB-231 (Figure 2B) cell lines.

Ferritin contains a superparamagnetic ferrihydrite $\left(5 \mathrm{Fe}_{2} \mathrm{O}_{3} \cdot 9 \mathrm{H}_{2} \mathrm{O}\right)$ crystal that increases the transverse NMR 
relaxation rate $\left(\mathrm{R}_{2}\right)$ of solvent water protons, causing a negative contrast in the corresponding MR images [30]. In order to assess whether native Ferritin can be exploited as a natural MRI probe for CSC detection, $\mathrm{T}_{2}$-weighted MR images were acquired following Ferritin incubation on both MDA-MB-231 and TUBO cells and their derived tumorspheres. Figure 2C shows that tumorspheres incubated with Ferritin displayed a markedly lower signal intensity when compared to untreated tumorspheres, while only small changes in signal intensity (SI) were observed in differentiated cells incubated in the absence or in the presence of Ferritin, respectively (Supplementary Material, Supplementary Table S1). Since spheroid diameter range is between 80 and $120 \mu \mathrm{m}$, TUBO-derived tumorspheres incubated 24 hours with $0.52 \mu \mathrm{M}$ Ferritin were detectable as isolated spots after their dispersion in agar (Figure 2D). Altogether, these data show that Ferritin-based contrast agents may be exploited for the MRI detection of CSC. The specificity of the uptake was assessed by carrying out a competition study by incubating cells for 24 hours in the presence of an excess of native Apoferritin. In both MDA-MB-231- and TUBO-derived tumorspheres, the iron uptake measured by ICP-MS decreased of about $60 \%$ and $66 \%$, respectively, confirming that iron uptake was mediated by Ferritin specific receptors. To ensure that the uptake of Ferritin by CSC is specifically mediated by SCARA5, we assessed the effect of SCARA5 silencing on the ability of cells to internalize Ferritin. TUBO-derived

A

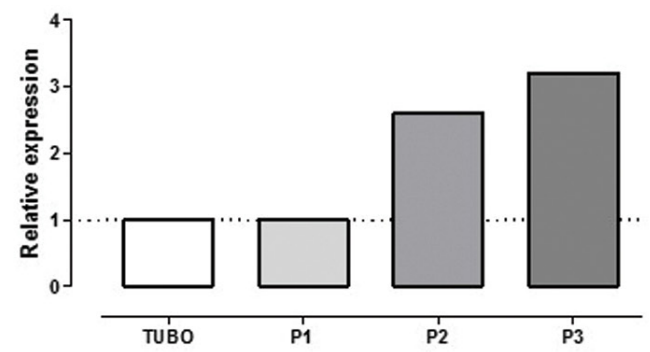

B

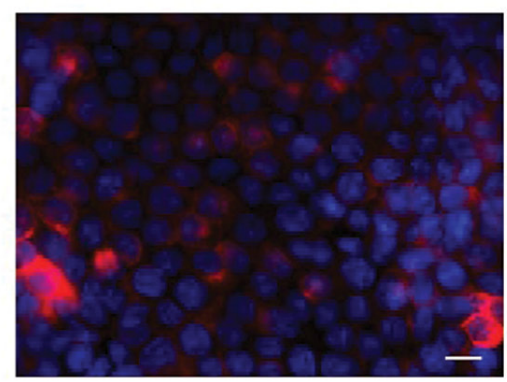

E

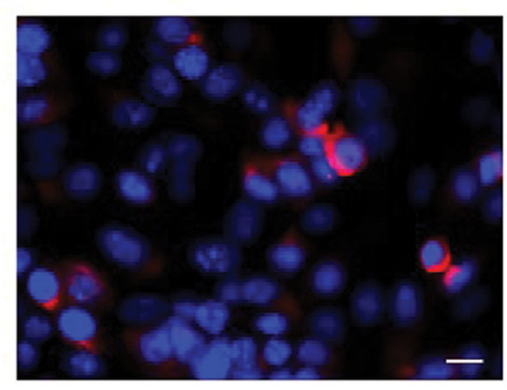

C

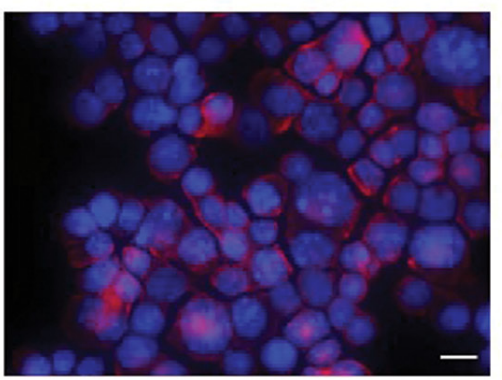

F

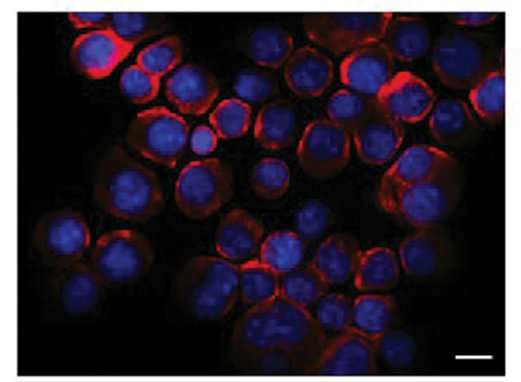

D

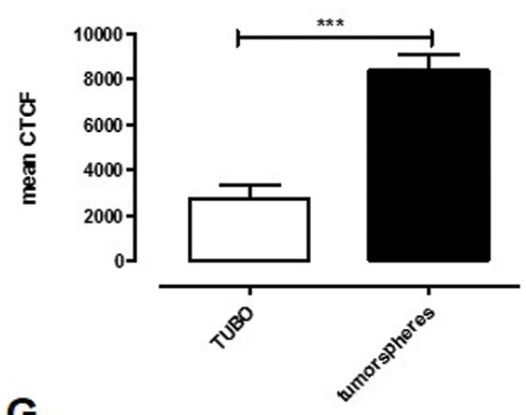

G

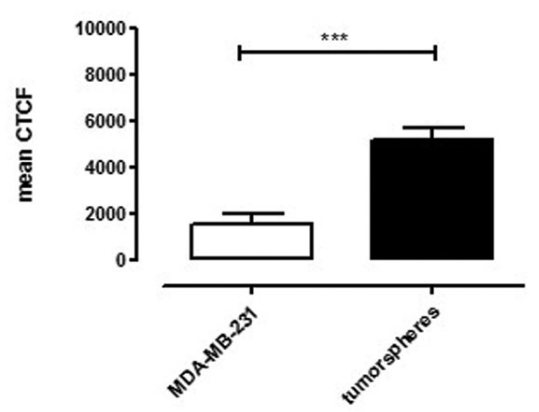

Figure 1: SCARA5 expression is upregulated in tumorspheres. A. Relative transcript expression level of SCARA5 in TUBO cells and in three different tumorsphere passages. B, E. Representative images of TUBO and MDA-MB-231 cells or of $\mathbf{C}, \mathbf{F}$. their derived tumorspheres stained with an anti-SCARA5 mAb (red). Nuclei were counterstained with DAPI (blue). Scale bar, $20 \mu \mathrm{M}$. D, G. Graphs represent the mean \pm SEM of the corrected total cell fluorescence (CTCF), calculated on at least 100 cells per sample as a quantification of SCARA5 expression in TUBO and MDA-MB-231 cells or in their derived tumorspheres. 
A

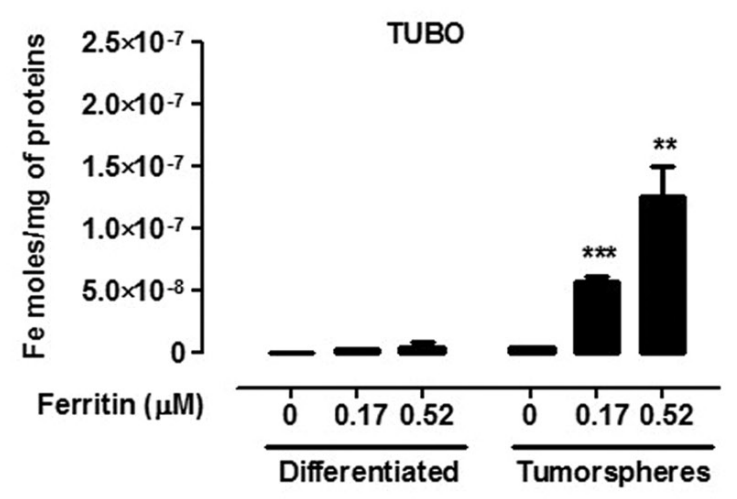

C

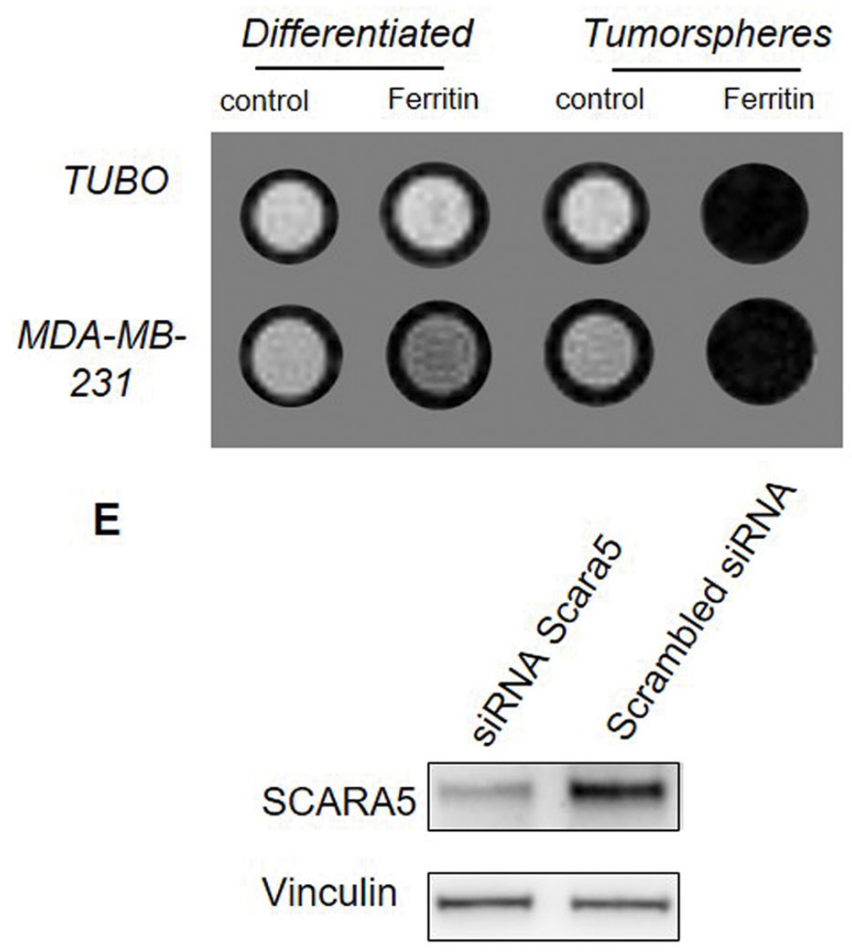

B

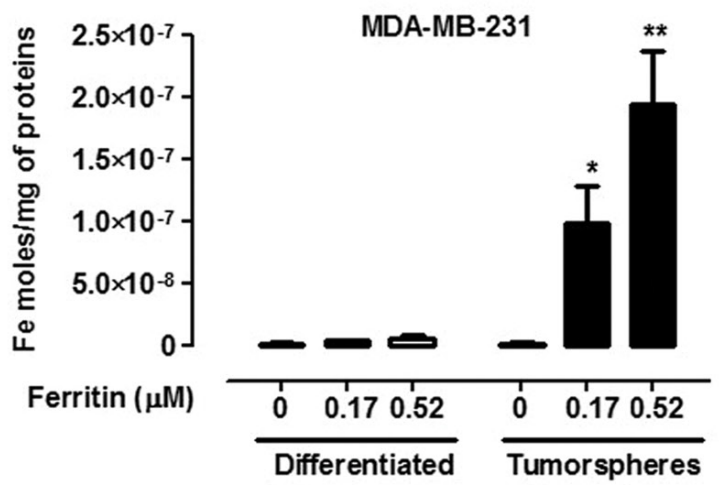

D
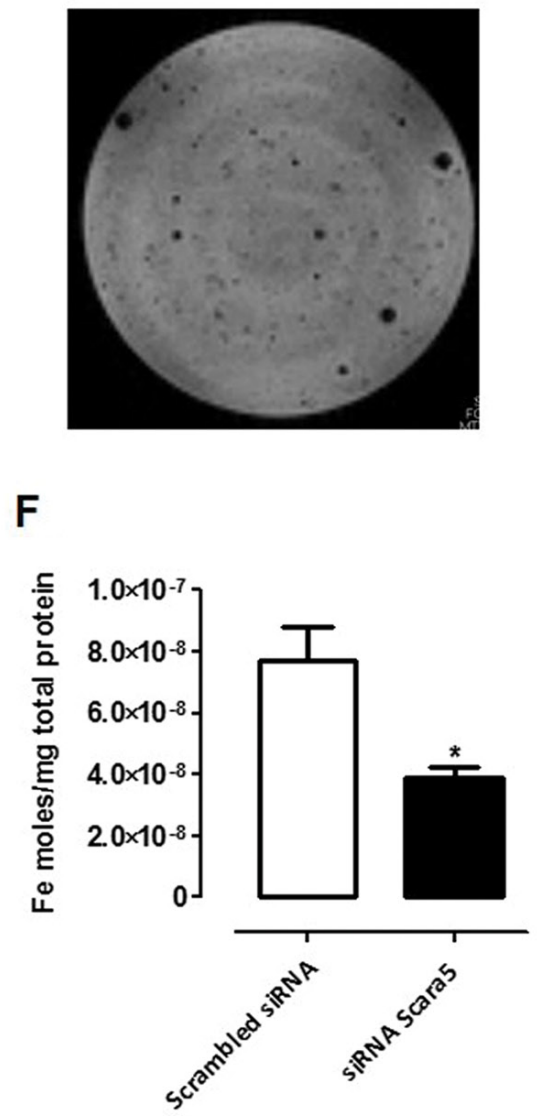

Figure 2: CSC display a higher Ferritin uptake than differentiated cells. A, B. ICP-MS determination of the intracellular iron content of TUBO (A) and MDA-MB-231 (B) cells and their derived tumorspheres cultured for 24 hours with or without Ferritin 0.17 and $0.52 \mu \mathrm{M}$. Graphs show the mean \pm SEM of internalized iron moles every $\mathrm{mg}$ of cell proteins from 3 independent experiments. C. A representative $\mathrm{T}_{2}$-weighted RARE MR image of an agar phantom containing TUBO and MDA-MB-231 cells (both differentiated and tumorspheres) incubated or not for 24 hours with L-Ferritin $0.52 \mu \mathrm{M}$. D. MRI of TUBO tumorspheres dispersed in agar. Each hypo-intense spot corresponds to the signal arising from one tumorsphere. E, F. TUBO derived tumorspheres were transfected with a siRNA to SCARA5 or a scrambled siRNA, and 48 hours after incubated with L-Ferritin for additional 24 hours. (E) Representative immunoblot of SCARA5 expression 48 hours after cell transfection. Vinculin expression was used as internal control. (F) Graph showing mean \pm SEM of iron moles every mg of cell proteins, evaluated by ICP-MS, from 3 independent experiments. * $\mathrm{p}<0.05$, Student's $t$ test. 
tumorspheres were incubated with a siRNA specific to SCARA5 or with a negative control scrambled siRNA, and the levels of SCARA5 protein were analyzed 24, 48 and 72 hours after transfection. SCARA5 transcript silencing (Supplementary Material, Supplementary Figure $\mathrm{S} 1)$, led to a threefold decrease in SCARA5 protein level 48 hours after transfection, and this was maintained for the subsequent 24 hours (Figure 2E, Supplementary Figure S1). Hence, 48 hours after transfection, cells were incubated with $0.52 \mu \mathrm{M}$ Ferritin for 24 hours. As shown in Figure 2F, the amount of iron internalized by cells treated with siRNA to SCARA5 was significantly lower than that internalized by control cells, thus confirming that SCARA5 mediates Ferritin uptake in breast CSC.

In order to further confirm that breast tumorspheres take up more Ferritin than their differentiated counterpart, Apoferritin was labelled with FITC on its external surface (hereafter referred to as APO-FITC), and TUBO cells and their derived tumorspheres were incubated for 24 hours in the presence of APO-FITC. Flow cytometric analysis revealed that APO-FITC was internalized by both TUBO and tumorspheres, but its intake in tumorspheres was significantly higher, as demonstrated by their enhanced mean fluorescence intensity (MFI, Figure 3A) when compared to TUBO cells. Of note, all cells expressing the stem cell marker Sca-1 [8], only present in tumorspheres, displayed a higher APO-FITC uptake than the remaining cells (Figure 3B, Sca1 ${ }^{+}$APO-FITC ${ }^{\text {high }}$ cells in the red boxes), suggesting that Ferritin uptake is higher in CSC than in more differentiated cells. Similarly, a small amount of APO-FITC was internalized in MDA-MB-231 differentiated cells, while its internalization in their derived tumorspheres was significantly higher (Figure 3C).

\section{Uptake and intracellular distribution of rhodamine isothiocyanate labelled apoferritin (Apo-Rhod)}

In order to perform a dose-response study using low Apoferritin concentrations, thus evaluating its affinity to SCARA5 receptors, Apoferritin was conjugated on its external surface with Rhodamine isothiocyanate (hereafter referred to as APO-Rhod), and incubated 24 hours with TUBO, MDA-MB-231 and their derived tumorspheres. Figure 4A shows the amount of APO-Rhod taken up by cells, determined using a calibration curve performed with a Rhodamine standard solution. These results confirm that the amount of internalized APO-Rhod was significantly higher in tumorspheres than in differentiated cells even at nanomolar concentrations, which is an indication of the high affinity of Apoferritin for SCARA5 receptor.

In order to assess whether the presence of $15 \%$ of $\mathrm{H}$-chains in the horse spleen Ferritin used in this study can mediate its uptake through $\mathrm{H}$-Ferritin receptors, a further competition study was carried out by incubating both MDA-MB-231 and TUBO tumorspheres for 24 hours with APO-Rhod in the presence of a 10-fold excess of recombinant $\mathrm{H}-$ Ferritin $[31,32]$. The $\mathrm{H}-$ Ferritin excess did not affect the amount of APO-Rhod internalized in both MDA-MB-231 and TUBO tumorspheres (Figure 4B) thus excluding the involvement of $\mathrm{H}$-Ferritin receptors in the internalization of Horse spleen Apoferritin.

To explore the underlying internalization mechanism, APO-Rhod was incubated with TUBOderived tumorspheres for fluorescence microscopy observation (Figure 4). Cells were then stained either for the early endosomal marker EEA-1 (panels C-F) or for the lysosomal marker LAMP-1 (panels G-L) to observe the process of APO-Rhod cellular trafficking. Two hours after incubation, APO-Rhod was located in the cytoplasm of most cells where it colocalized with early endosomes, as shown in panel F. LAMP-1 was distributed throughout the cytoplasm and the codistribution of APO-Rhod with LAMP-1 signal was circumscribed to the granular regions in the cytoplasm of some cells (Figure 4, panel L). These observations indicate that APO-Rhod was delivered to early endosomes after internalization. In the canonical receptor-mediated endocytosis pathway, early endosomes gradually mature to became late endosomes that then converge in lysosomes [33]. Most probably, APO-Rhod partly follows this path after internalization, since it colocalizes with the lysosomal marker LAMP-1.

\section{Uptake of apoferritin loaded with Gd-HPDO3A MRI contrast agent and Curcumin (Gd-APO- curcumin)}

Despite its structural stability under physiological conditions, Ferritin displays a $\mathrm{pH}$-dependent de-assembly, which can be exploited to load it with both therapeutic agents and imaging probes. Indeed, there are many examples where Ferritin nanoarchitecture was broken down in acidic environment and restored by retuning the $\mathrm{pH}$ to 7.4 , after the entrapment of the desired solutes in its inner cavity $[22,34]$. Using this procedure, Curcumin and the commercially available MRI contrast agent GdHPDO3A, neutral and safe even at high concentrations [35], were entrapped in the Apoferritin cavity (Figure 5A). Curcumin was selected as therapeutic agent since it has been reported to exhibit anti-oxidant, anti-inflammatory, anti-microbial and anti-cancer activity in vitro and in animal models of several diseases, such as acute hepatitis, acute ileitis, neuroinflammation, ischaemia and cancer [36]. Its anti-cancer activity is operated through modulation or inhibition of multiple molecular pathways [37]. Furthermore, during the past few years, a number of studies have suggested that Curcumin may have direct or indirect influence on CSC self-renewal pathways, including Wnt/b-catenin, sonic hedgehog, and Notch [38-40]. Another important property is that, unlike other known chemotherapeutic compounds, Curcumin does not 
cause any damage to normal cells [41]. Since SCARA5 is also expressed in the liver, the observation that Curcumin had no effect on normal rat hepatocytes [41] is essential to promote its use as a valid chemopreventive and chemotherapeutic agent for CSC using Apoferritin as a delivery platform. Furthermore, loading Curcumin into its protein cavity improves Curcumin bioavailability and stability under physiological conditions, maintaining its peculiar pharmacological properties [22]. Curcuminloaded Apoferritin (APO-Curcumin) stability was assessed spectrophotometrically by measuring absorbance decrease at $430 \mathrm{~nm}$. In the first 2 hours the absorbance showed a small decrease $(15 \%)$, then it remained essentially constant during the entire experimental time (48 hours). Without Apoferritin, more than $90 \%$ of Curcumin decomposed rapidly (30 minutes) in buffer at neutral $\mathrm{pH}$ as already reported by Zebib et al. [42].

Using the protocol described above, the number of molecules that remained in Apoferritin inner cavity after the dissociation/reassociation procedure was $228 \pm 48$ and $9.6 \pm 2$ for Curcumin and Gd-HPDO3A, respectively. GdAPO-Curcumin is stable for at least $48 \mathrm{~h}$ as assessed by
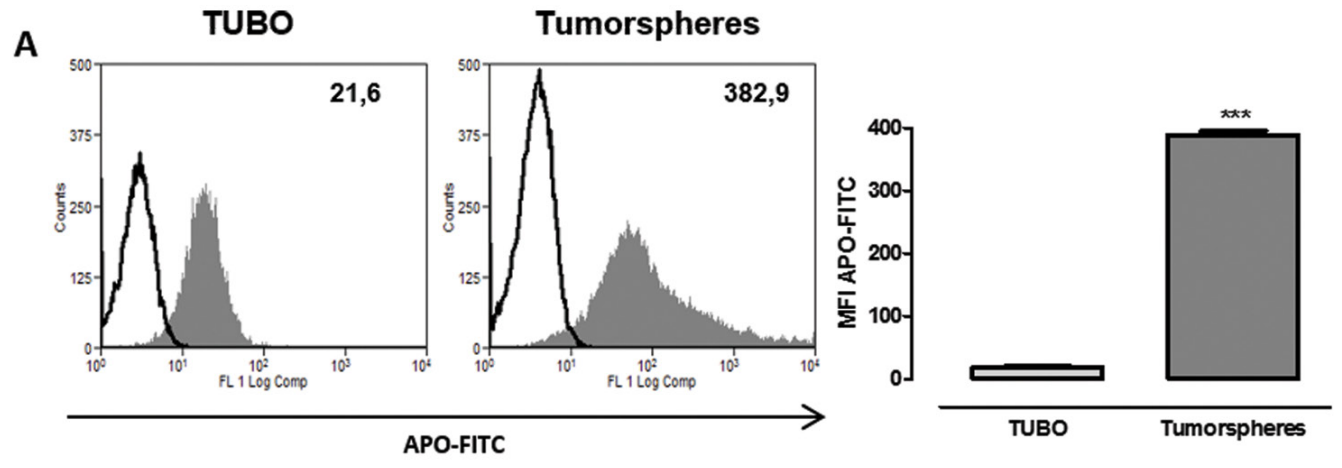

\section{B}
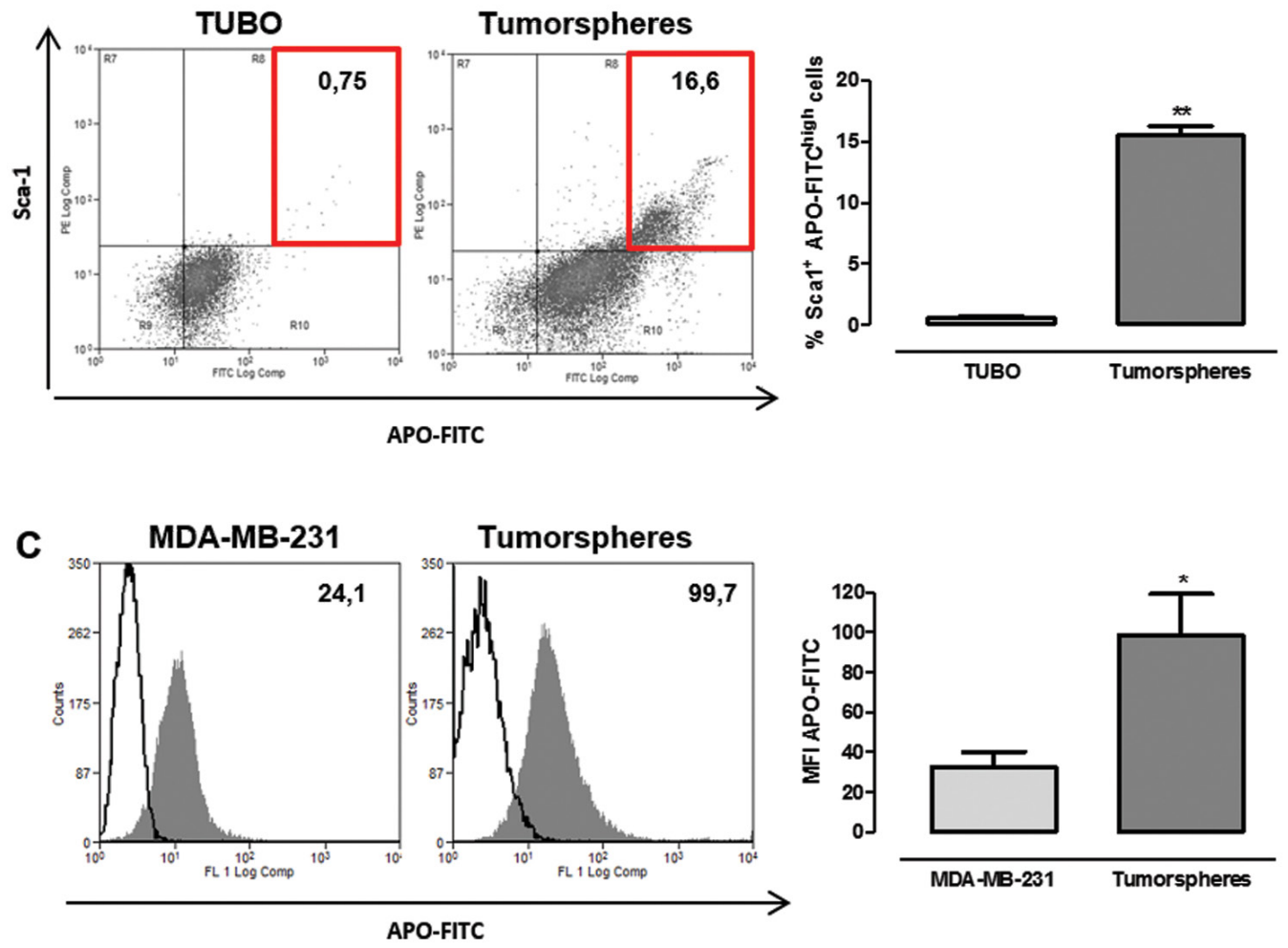

Figure 3: CSC display a higher APO-FITC uptake than differentiated cells. FACS analysis of TUBO and MDA-MB-231 cells and their derived tumorspheres cultured with or without APO-FITC for 24 hours. A, C. Representative histograms of untreated (open histograms) or APO-FITC treated (gray histograms) TUBO (A) and MDA-MB-231 (C) cells and tumorspheres. Numbers show mean fluorescent intensity (MFI), the graphs show the mean \pm SEM of APO-FITC MFI observed in cells and tumorspheres from 4 independent experiments. B. Representative dot plots of APO-FITC and Sca-1 expression in TUBO and tumorspheres. Numbers in quadrants show the percentage of APO-FITC ${ }^{\text {high }} \mathrm{Sca}^{+}{ }^{+}$cells (evidenced by the red boxes), the graph shows the mean $\pm \mathrm{SEM}$ of the percentage of APO-FITC ${ }^{\text {high }}$ Sca- $1^{+}$cells in TUBO and tumorspheres from 4 independent experiments. ${ }^{*} P<0.05,{ }^{* *} P<0.01,{ }^{* * *} P<0.001$; Student's $t$ test. 
measuring absorbance at $430 \mathrm{~nm}$ [17]. The $\mathrm{T}_{1}$-weighted MRI image, recorded after 24 hours incubation in the presence of Gd-APO-Curcumin $(2.7 \mu \mathrm{M})$, showed that the signal arising from labelled tumorspheres was clearly hyperintense compared to the untreated control tumorspheres for both cell lines (Figure 5B and Supplementary Materials, Supplementary Table S2). On the contrary, no significant signal enhancement was observed in Gd-APO-Curcumin-treated differentiated cells when compared to untreated cells. These observations were confirmed by the significantly higher amount of $\mathrm{Gd}$ taken-up by tumorspheres, measured by ICP-MS (Figure 5C). On the basis of the ICP-MS Gd measurements, it was possible to calculate the intracellular Gd, and consequently Curcumin, concentrations. In fact, since the Curcumin/ Gd ratio in the Apoferritin preparation was 16, estimated intracellular Curcumin concentrations of 1400 and 460 $\mu \mathrm{g} / \mathrm{g}$ were obtained for MDA-MB-231 and TUBO derived tumorspheres, respectively.

\section{APO-Curcumin induces cell death and reduces self-renewal of CSC}

In order to seek whether APO-Curcumin is able to induce inhibitory effects on CSC survival and selfrenewal, TUBO cells and their derived tumorspheres where incubated in the presence or absence of APOCurcumin for different time intervals, and cell death was evaluated by citofluorimetric analysis with Annexin $\mathrm{V}$ and propidium iodide staining. As shown in Figure 6A, APOCurcumin did not induce cell death in TUBO cells, while progressively caused cell death in tumorspheres when compared to untreated cells. Of note, APO-Curcumin was more efficient than free Curcumin in inducing cell
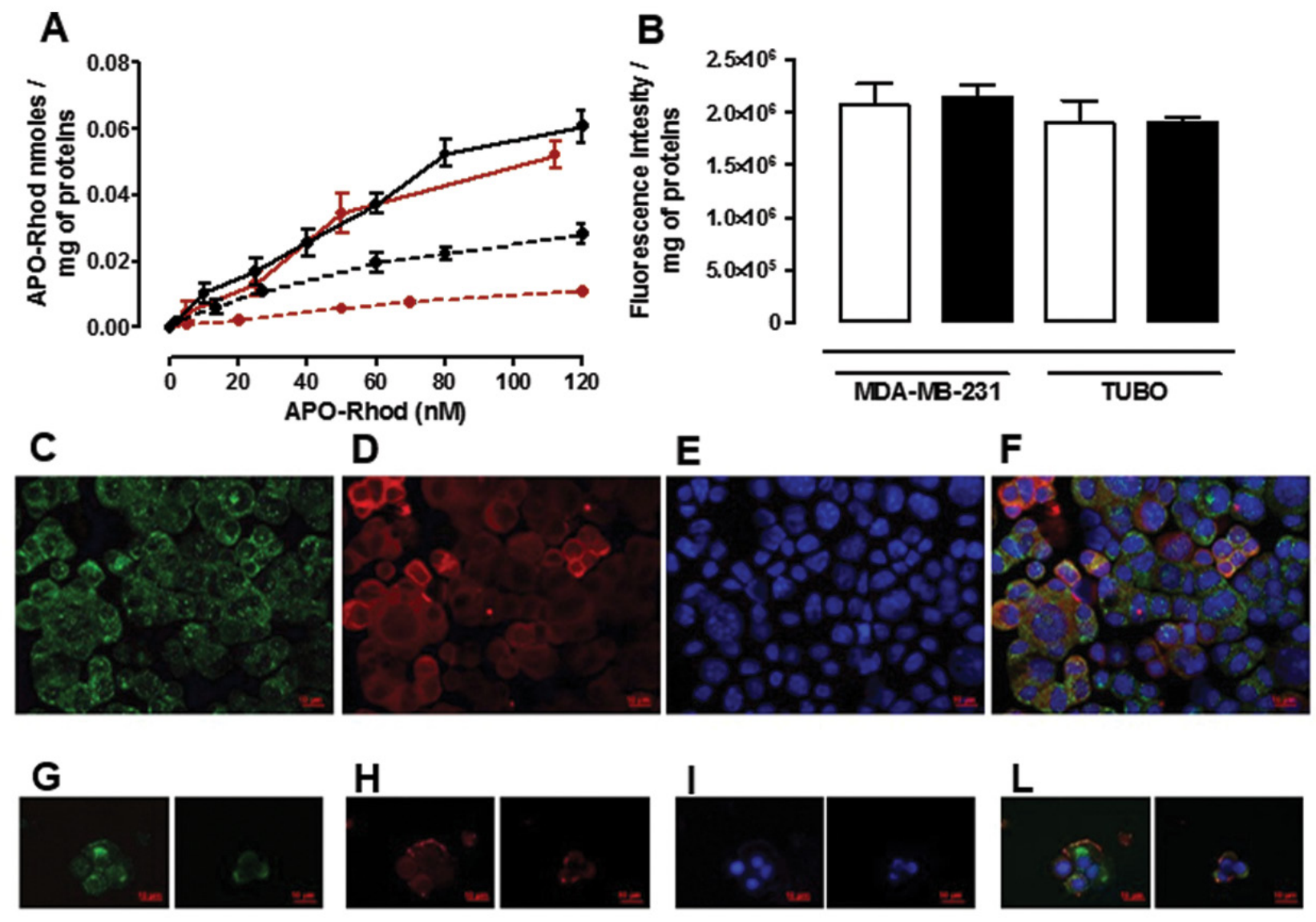

Figure 4: Uptake and intracellular distribution of Apo-Rhod. A. Dose-response curve obtained by incubating different concentrations of APO-Rhod 24 hours with TUBO (red dotted line), MDA-MB-231 (black dotted line) and their derived tumorspheres (red and black continuous lines respectively). B. Apo-Rhod concentrations were determined by measuring fluorescence (ex/em $555 / 575 \mathrm{~nm}$ ) on cytosolic extracts and were normalized to the cell proteins. Fluorescence intensity (au) of cytosolic extracts of TUBO and MDA-MB-231 derived tumorspheres, incubated in the absence (white bars) or in the presence of a 10 fold excess of H-Apoferritin. C-L. Representative images of TUBO derived tumorspheres incubated for 2 hours at $37^{\circ} \mathrm{C}$ with APO-Rhod (red, D and H) and stained with an anti-EEA1 antibody (green, C) or an anti-LAMP-1 antibody (green, G). Nuclei were counterstained with DAPI (blue, E and I). The co-localization between APO-Rhod and EEA1 or LAMP-1 is shown in the merged images of panels F and L, respectively. Scale bar, $10 \mu \mathrm{M}$. 
death in tumorspheres (Figure 6B). This enhanced effect was not due to any toxicity of the protein itself, since treatment with Apoferritin did not induce cell death in either TUBO or tumorspheres (Figure 6B), but likely to the ability of APO-Curcumin to enhance Curcumin uptake by CSC. APO-Curcumin and Curcumin affected
A

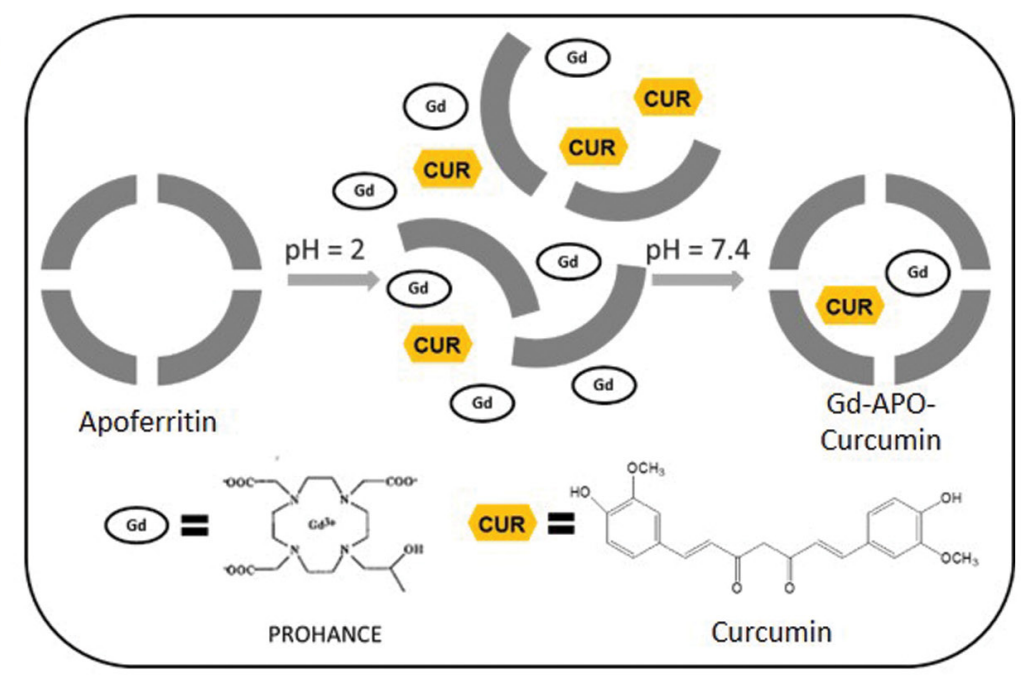

B

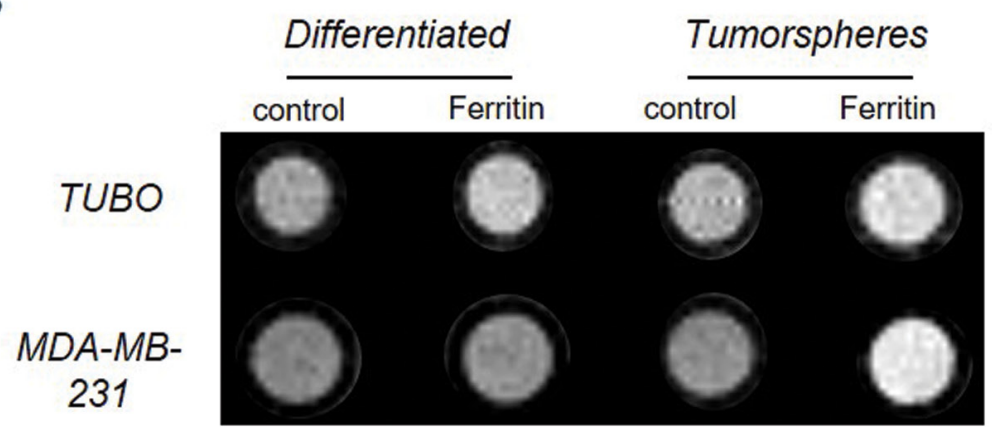

C

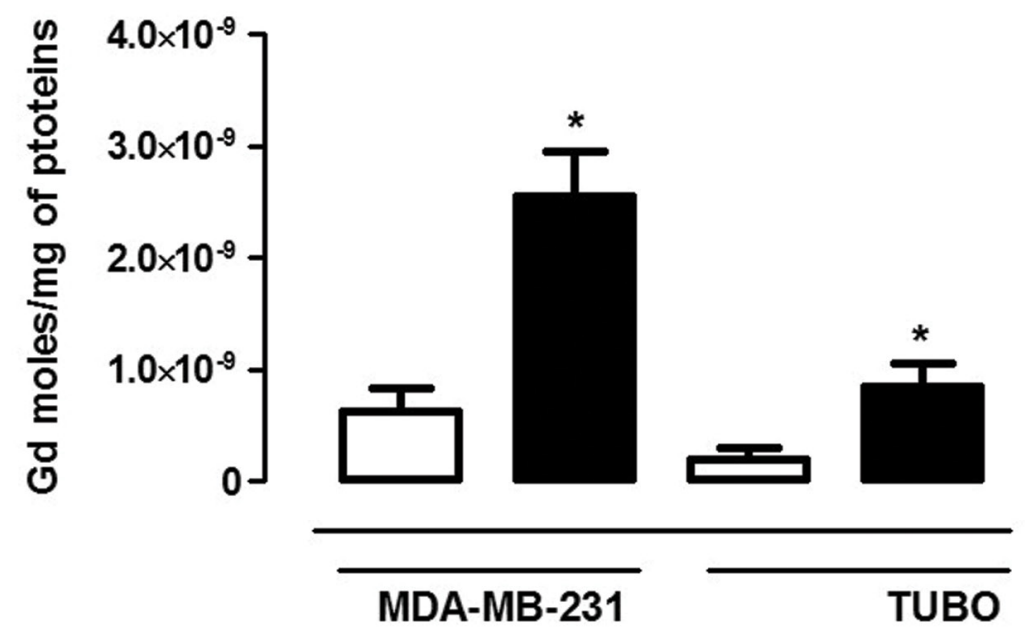

Figure 5: Gd-APO-Curcumin uptake is higher in CSC than in differentiated cells. A. Schematic representation of Gd-APOCurcumin preparation. B. A representative $\mathrm{T}_{1}$-weighted spin echo image of an agar phantom containing TUBO and MDA-MB-231 (both differentiated and tumorspheres) cells incubated or not for 24 hours with Gd-APO-Curcumin $(2.7 \mu \mathrm{M}$ in protein). C. Mean $\pm \mathrm{SEM}$ of internalized Gd moles every mg of cell proteins from 3 independent experiments, measured by ICP-MS as in (B). *P<0.05; Student's $t$ test. 
not only tumorsphere viability, but also CSC self-renewal capacity as shown by a decreased ability of treated cells to re-generate tumorspheres, which on the contrary was not hindered by treatment with Apoferritin alone (Figure 6C).
None of these compounds altered TUBO cell morphology (Figure 6D, upper panels), while tumorspheres cultured in presence of Curcumin and APO-Curcumin were smaller than those cultured in medium alone or with Apoferritin

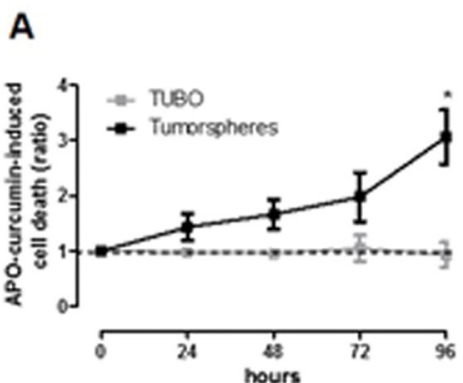

D
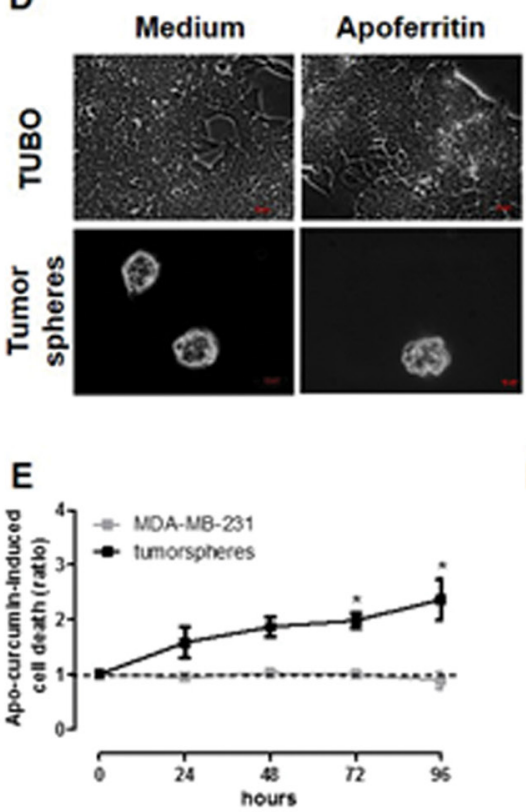

H

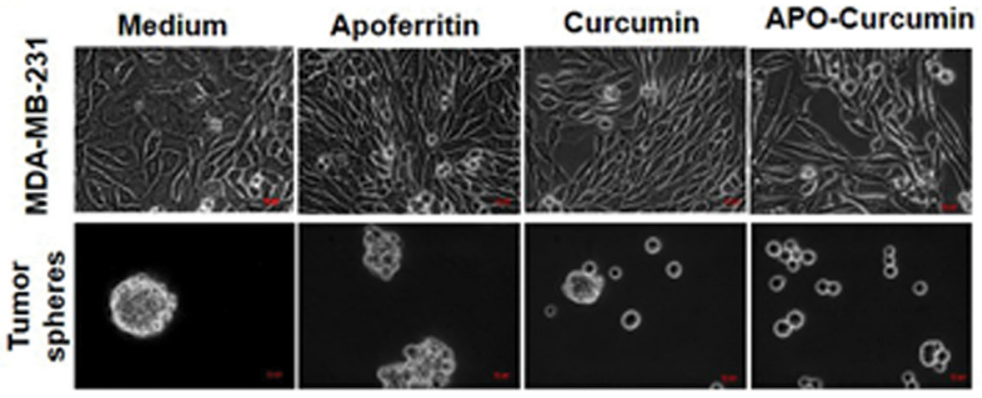

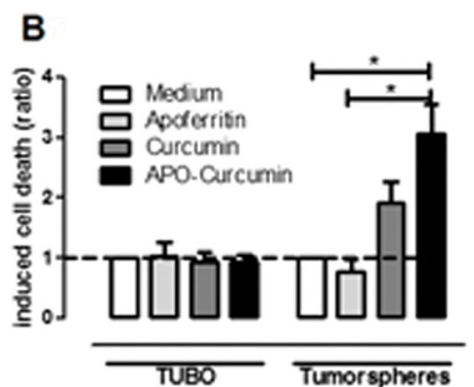
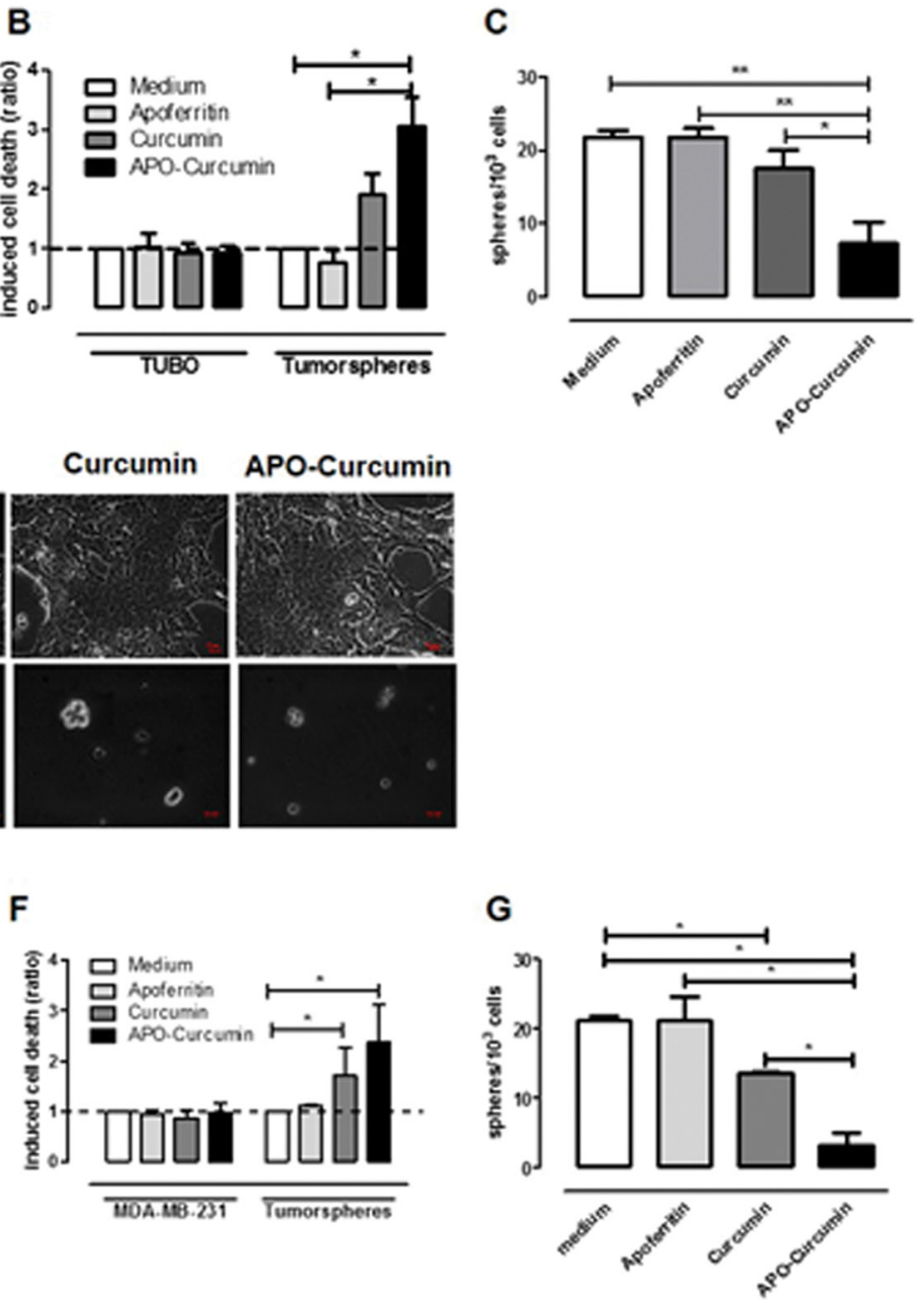

Figure 6: APO-Curcumin induces cell death and reduces self-renewal in tumorspheres. TUBO A-D. and MDA-MB-231 EH. cells were cultured as monolayer or tumorpsheres in presence of Apoferritin, APO-Curcumin or Curcumin. (A, E) After 24, 48, 72 or 96 hours, cells were stained with Annexin-V and PI and analyzed by FACS. The graphs show means \pm SEM of the ratio of dead cells between APO-Curcumin-treated and untreated cells, calculated as described in M\&M. (B, F) Graphs showing means \pm SEM of the ratio of the percentage of dead cells present in samples treated for 96 hours with Apoferritin, APO-Curcumin or Curcumin in comparison to untreated samples. $(C, G)$ The graphs show means \pm SEM of the number of spheres generated every $10^{3}$ cells plated in the absence or presence of Apoferritin, APO-Curcumin or Curcumin. (D, H) Representative images of differentiated (upper panels) or tumorspheres (lower panels) cultured for 96 hours with the different treatments. Magnification 40X, scale bars $100 \mu \mathrm{m}$. All experiments were repeated at least 4 times. $* P<0.05 ; * * P<0.01$; Student's $t$ test. 
(Figure 6D, lower panels). The ability of APO-Curcumin to target CSC and decrease their survival and self-renewal is not restricted to the TUBO model, since similar results were obtained with MDA-MB-231 cells (Figure 6E$6 \mathrm{H})$. While the increased effect of APO-Curcumin on tumorspheres compared to differentiated cells can be explained by tumorspheres' increased expression of SCARA5 receptor, the reason why even free Curcumin $(50 \mu \mathrm{M})$ affects tumorspheres but not differentiated cells is more speculative. It has been shown by others $[38,43]$ that Curcumin acts primarily on undifferentiated stem and progenitor cells rather than on more differentiated cells from mammary tissue and breast cancer cell lines. This effect appears to be mediated, at least in part, by the inhibition of intracellular signalling pathways that are upregulated in CSC [38]. It is therefore highly likely that a similar mechanism of action occurs also in our model.

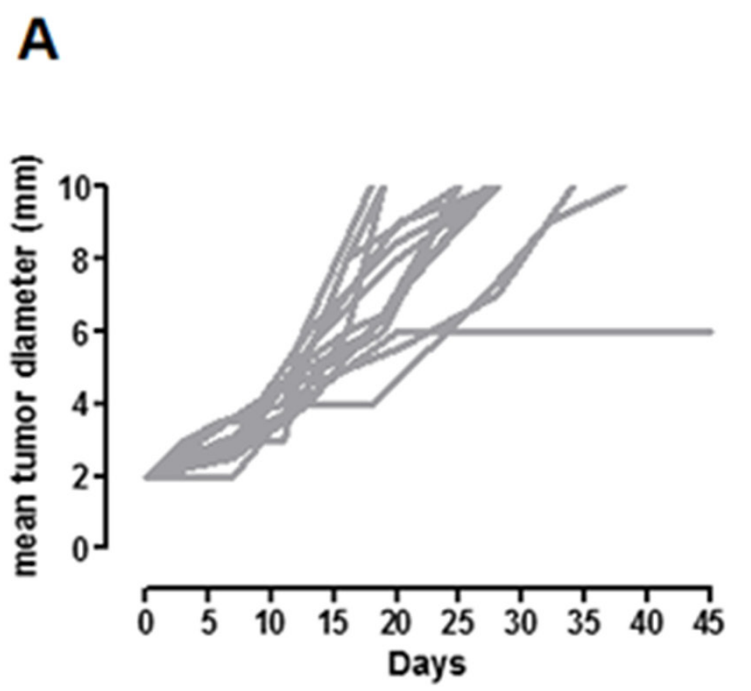

C

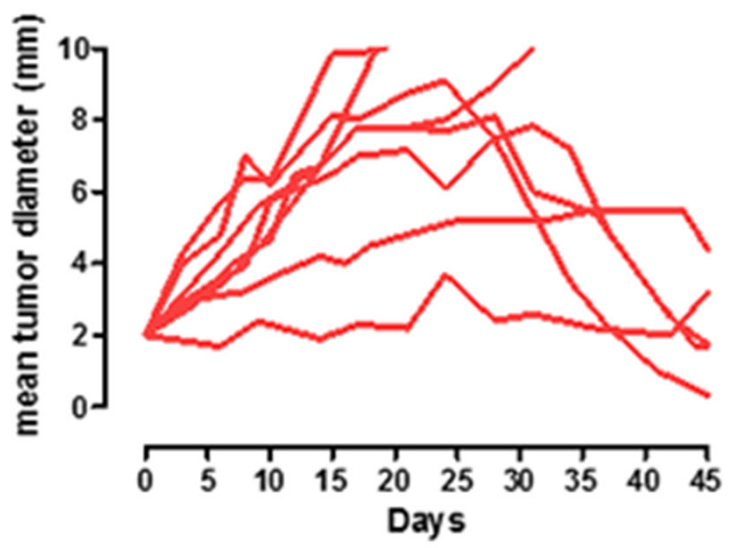

\section{APO-Curcumin inhibits breast cancer growth in vivo}

To explore the efficacy of APO-Curcumin on established tumors in vivo, we set up a model that consists in the subcutaneous (s.c.) implantation of TUBOderived tumorspheres into syngeneic BALB/c mice. After implantation, these cells maintain a prevalent CSC phenotype, as demonstrated by the presence of an higher level of both $\mathrm{CD}_{4} 4^{+} / \mathrm{CD} 24^{-}$and Aldefluor ${ }^{+}$expressing cells when compared to tumors generated by TUBO cells (Supplementary Material, Supplementary Figure S2). When tumors reached $2 \mathrm{~mm}$ mean diameter, mice were treated with APO-Curcumin or Apoferritin (10 mg/Kg of Curcumin and $53 \mathrm{mg} / \mathrm{Kg}$ of protein) intravenously (i.v.) every 3 days, or left untreated (Figure 7). The extremely low Curcumin water solubility prevents its direct i.v.

B

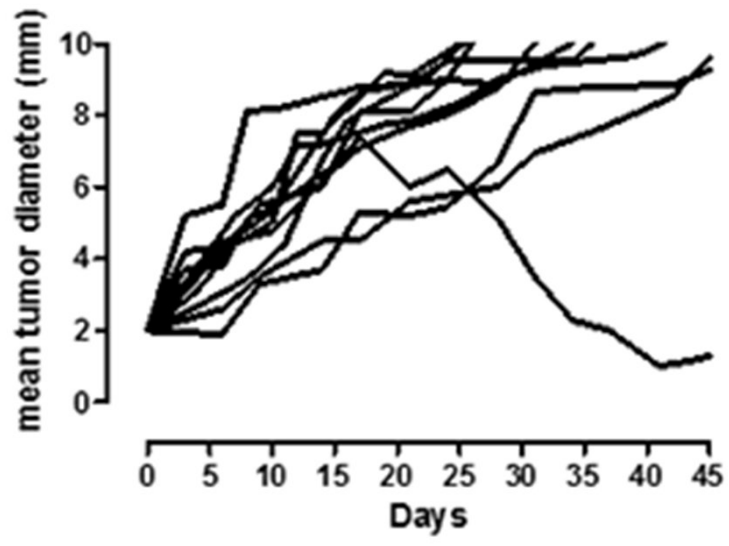

D

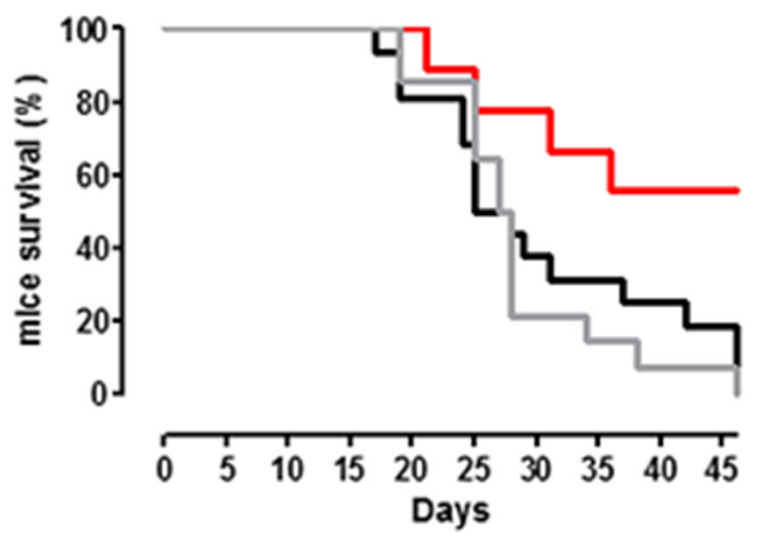

Figure 7: APO-Curcumin treatment delays CSC-induced tumor growth in vivo. A-C. BALB/c mice were challenged s.c. with TUBO-derived tumorspheres and left untreated (A, gray lines) or treated i.v. with Apoferritin (B, $53 \mathrm{mg} / \mathrm{Kg}$; black lines) or APO-Curcumin (C, $10 \mathrm{mg} / \mathrm{Kg}$ of Curcumin and $53 \mathrm{mg} / \mathrm{Kg}$ of protein; red lines) every 3 days, starting when their tumor reached $2 \mathrm{~mm}$ mean diameter. Each line depicts the growth of a single tumor. D. Kaplan-Meier survival curves. APO-Curcumin vs Apoferritin treated mice: $P=0.0189$; Apoferritin treated mice vs Untreated mice: $P=0.5$; APO-Curcumin treated mice vs Untreated mice: $P=0.0054$, Log-rank (Mantel-Cox) Test. Data were cumulated from two independent and concordant experiments. 
Table 1: Percentage of mice whose tumor regressed (responders) or grew (non responders) in the three groups

\begin{tabular}{lccc}
\hline & APO-Curcumin & Apoferritin & Untreated \\
\hline Responders & $5 / 8(62.5 \%)$ & $1 / 10(10 \%)$ & $0 / 11(0 \%)$ \\
Non Responders & $3 / 8(37.5 \%)$ & $9 / 10(90 \%)$ & $11 / 11(100 \%)$ \\
\hline
\end{tabular}

Data were cumulated from two independent and concordant experiments. APO-Curcumin vs Apoferritin treated mice: $P=0,043$; Apoferritin treated mice vs Untreated mice: $P=0.47$; APO-Curcumin treated mice vs Untreated mice:

$P=0.0048$, Chi square test.

administration without exploiting a nanoformulation/ emulsion, therefore it was not possible to insert a Curcumin-treated group. Whereas virtually all tumors grew progressively in untreated or Apoferritin-treated mice (Figure 7A, 7B and Table 1), they did not progress or eventually regressed in about $60 \%$ of APO-Curcumin treated mice (Figure 7C and Table 1).

Considering the animal survival, whereas all untreated mice died at the end of the experiment, about $60 \%$ of APO-Curcumin treated mice were still alive, compared to only $10 \%$ in the Apoferritin treated group (Figure 7D; $P=0.018$, Chi square test), suggesting that Curcumin-loaded Apoferritin may represent a promising tool for breast cancer treatment.

In order to verify that APO-Curcumin is not toxic to the liver, being SCARA 5 also expressed on hepatocytes, histological assessment of liver damage was carried out at the end point of the treatment. Periportal hepatocytes from both Apoferritin and APO-Curcumin treated animals did not show any injury but only a moderate degree of vacuolar degeneration, which is compatible with lysosomal compartment enlargement due to the endocytic uptake of the nanodevice (Supplementary Material, Supplementary Figure S3).

In conclusion, in this work it was shown that both human and mice breast CSC display an increased L-Ferritin uptake capability compared to their more differentiated counterparts. This is mediated at least in part by the upregulation of the L-Ferritin receptor SCARA5. These results open new horizons in the design of targeting strategies for the eradication of CSC, which are usually highly resistant to conventional chemo- and radiotherapy. Indeed, the increased L-Ferritin uptake can be exploited for the delivery of Curcumin to CSC, as the loading of Curcumin into L-Apoferritin does not cause any reduction of its affinity for the SCARA5 receptor. Of note, loading Curcumin into Apoferritin not only enables specific targeting to SCARA5-expressing cells, and thus to breast CSC, but also improves Curcumin bioavailability, opening up the possibility of in vivo treatments. Our in vivo results highlight the therapeutic potential of APO-Curcumin. However, to improve the clinical translatability of this approach, further experiments are needed in order to explore the synergistic effect of APO-
Curcumin and classical chemotherapy. In this way, the elimination of CSC, exerted by APO-Curcumin, could be accompanied by the eradication of more differentiated tumor cells, leading to overcome tumor resistance and reduce recurrence. The relatively low sensitivity of MRI hampers the use of Gd-APO-Curcumin for the in vivo detection of CSC that represent a very low proportion of the tumor mass. However, this important objective might be achieved by developing more sensitive Apoferritin based PET or optical probes, which could provide new theranostic tools to improve breast patient stratification and monitoring of their response to therapy.

\section{MATERIALS AND METHODS}

\section{Preparation of Gd-HPDO3A and Curcumin loaded Apoferritin (Gd-APO-Curcumin)}

Gd-HPDO3A (ProHance) was kindly provided by Bracco Imaging S.p.A. Apoferritin, Ferritin (from horse spleen), Curcumin and all other chemicals were purchased from Sigma-Aldrich. H-Ferritin was kindly provided by Paola Turano (Center for Magnetic Resonance, University of Florence Italy) and prepared as described in [31, 32]. The loading of Curcumin and Gd-HPDO3A in the iron free Apoferritin cavity was carried out as described previously [22]. Briefly, the dissociation of Apoferritin into its subunits was done by lowering the $\mathrm{pH}$ of the $4.1 \times 10^{-6} \mathrm{M}$ protein solution $(8 \mathrm{~mL})$ to $\mathrm{pH} 2$ using $1 \mathrm{M}$ $\mathrm{HCl}$ and maintaining this low $\mathrm{pH}$ for about 15 minutes. Afterwards, $50 \mu \mathrm{L}$ of a Curcumin solution in DMSO (200 $\mathrm{mg} / \mathrm{mL}$ ) and $2 \mathrm{~mL} 0.5 \mathrm{M}$ Gd-HPDO3A were added to the Apoferritin solution. Then the $\mathrm{pH}$ was adjusted to 7.4 using $1 \mathrm{M} \mathrm{NaOH}$. The resulting solution was stirred at room temperature for 2 hours, centrifuged, purified by gel filtration (Superdex G25 Column, Amersham) and dialysis. The solution was then concentrated using Vivaspin centrifugal concentrators (50 000 MWCO, SigmaAldrich). At the end of this process the concentrations of the protein and of Curcumin were measured by Bradford assay (using bovine serum albumin as a standard) and spectrophotometrically at $430 \mathrm{~nm}$ in ethanol, respectively. The final Gd concentration was determined by inductively 
coupled plasma mass spectrometry (ICP-MS) (Element-2; Thermo-Finnigan). Sample digestion was performed with $2 \mathrm{~mL}$ of concentrated $\mathrm{HNO}_{3}(70 \%)$ under microwave heating (Milestone MicroSYNTH Microwave Labstation).

\section{Cell lines}

MDA-MB-231 were purchased from LGC Standards and grown in DMEM (Invitrogen Corp.) supplemented with 10\% FBS (Sigma-Aldrich). TUBO cells [44] were generated from a spontaneous tumor of Her2/neu transgenic (BALB-neuT) mice [45] and were cultured in DMEM supplemented with $20 \%$ FBS. All cells were tested negative for mycoplasma by PCR assay and passaged in our laboratory for fewer than six months after their resuscitation. For tumorsphere generation, cells were detached and plated in ultra-low attachment flasks (SigmaAldrich) at $6 \times 10^{4}$ viable cells $/ \mathrm{mL}$ in mammosphere medium, as previously reported [8].

\section{Ferritin uptake experiments}

MDA-MB-231, TUBO cells and their derived tumorspheres were incubated with increasing concentrations of Ferritin, Gd-APO-Curcumin and Apoferritin 24 hours post seeding. After 24 hours of incubation, cells were washed three times with ice-cold PBS and detached with trypsin/EDTA (Sigma-Aldrich). Then cells were lysed by sonication using an ultrasonic probe device (30\% power). The Fe or Gd content in each cell line was determined by ICP-MS as described above. For MRI analysis (see below) cells were transferred into glass capillaries. The protein concentration was determined from cell lysates by the Bradford assay. The amounts of iron (measured by ICP-MS) internalized by cells were normalized to cell proteins concentration. $1 \mathrm{mg}$ of proteins measured on cell lysate correspond to $3.1 \pm 0.3 \times 10^{6}$ and $2.8 \pm 0.3 \times 10^{6}$ MDA-MB-231 differentiated cells and tumorspheres, respectively, and to $2.9 \pm 0.4 \times 10^{6}$ and $3.8 \pm 0.35 \times 10^{6}$ TUBO differentiated cells and tumorspheres, respectively.

\section{MRI}

All the MR images were acquired on a Bruker Avance 300 spectrometer (7 T) equipped with a Micro 2.5 microimaging probe (Bruker BioSpin). For in vitro determinations, glass capillaries containing $2 \times 10^{6}$ cells were placed in an agar phantom and MRI was performed using a standard $\mathrm{T}_{1}$-weighted multislice spinecho sequence $(\mathrm{TR} / \mathrm{TE} / \mathrm{NEX}=250 / 3.3 / 8, \mathrm{FOV}=1.2$ $\mathrm{cm}, \mathrm{NEX}=$ number of excitations; FOV $=$ field of view). $\mathrm{T}_{2}$-Weighted MRI images were obtained using a RARE sequence protocol (TR/TE/NEX = 5000/53/4; FOV= $1.2 \mathrm{~cm}$; MTX $128 \times 128)$. The $\mathrm{T}_{1} / \mathrm{T}_{2}$ relaxation times were calculated using a standard saturation recovery spin echo. The image of tumorspheres dispersed in agar were obtained using a 3D FLASH gradient echo (TR/TE/NEX $=3500 / 18 / 2 ; \mathrm{FOV}=1.14 \mathrm{~cm}$; MTX 128x128x128).

\section{APO-FITC and APO-rhodamine preparation}

$2 \mathrm{mg} / \mathrm{mL}$ Apoferritin solution in $0.1 \mathrm{M}$ sodium carbonate buffer at $\mathrm{pH} 9$ was prepared. Fluorescein isothiocyanate (FITC, Sigma-Aldrich) or Rhodamine isothiocyanate (Rhod) were dissolved in anhydrous DMSO at $1 \mathrm{mg} / \mathrm{mL}$. For each $\mathrm{mg}$ of protein, $50 \mu \mathrm{L}$ of dye (FITC or Rhod) solution were added slowly (in 5 $\mu \mathrm{L}$ aliquots) while stirring the protein solution. When all the required amount of dye solution had been added, the reaction mixture was incubated in the dark for 16 hours at $4^{\circ} \mathrm{C}$. Then $\mathrm{NH}_{4} \mathrm{Cl}$ was added to the solution to a final concentration of $50 \mathrm{mM}$, and it was incubated for other 2 hours at $4^{\circ} \mathrm{C}$. The obtained Apoferritin solution was purified from non-entrapped dye with gel filtration using a G25 sephadex column, followed by dialysis. After purification, the solution was characterized in terms of protein concentration using the Bradford assay. The dye concentration was determined by measuring fluorescence (Horiba FluoroMax-4 spectrofluorometer), in Triton 0.1\%, at $492 / 517 \mathrm{~nm}$ and $555 / 575 \mathrm{~nm}$ excitation/emission for FITC and Rhod, respectively.

\section{APO-Rhodamine uptake experiments}

MDA-MB-231, TUBO cells and their derived tumorspheres were incubated with increasing concentrations of APO-Rhod 24 hours post seeding. After 24 hours of incubation, cells were washed three times with ice-cold PBS and detached with trypsin/EDTA. Then cells were lysed by sonication using an ultrasonic probe device (30\% power) and rhodamine concentration in the cytosolic extracts was determined by measuring fluorescence as described in the previous paragraph. Competition study has been performed by comparing fluorescence intensity of TUBO and MDA-MB-231 tumorspheres incubated with APO-Rhod $25 \mathrm{nM}$ (protein concentration) in the presence and in the absence of H-Ferritin $250 \mathrm{nM}$ for 24 hours. Fluorescence intensity (expressed as arbitrary units) was normalized to the total protein cell content.

\section{Fluorescent microscopy}

For SCARA5 detection, $3 \times 10^{5}$ TUBO and MDAMB-231 cells were plated on glass coverslips and left to adhere overnight at $37^{\circ} \mathrm{C}$ in a $5 \% \mathrm{CO}_{2}$ incubator. $1 \times 10^{5}$ tumorsphere-derived cells were cytospinned (Cytospin 4, ThermoScientific) to glass slides. Then, cells were fixed with 4\% formaldehyde solution in PBS (Sigma-Aldrich) and stained with an anti-SCARA5 antibody (Thermo Scientific), as previously described [25]. To visualize the internalization of Apoferritin, $6 \times 10^{5}$ disaggregated 
TUBO-derived tumorspheres were incubated for 2 hours at $37^{\circ} \mathrm{C}$ with APO-Rhod $(0,47 \mu \mathrm{M}$ in Rhod and $0,073 \mu \mathrm{M}$ in Apoferritin). Cells were then washed twice with ice-cold PBS, cytospinned to glass slides, fixed in cooled methanol for $10 \mathrm{~min}$ and permeabilized in cooled acetone for $1 \mathrm{~min}$. Slides were then stained with antiEEA1 (Cell Signaling Technology) or with anti-LAMP-1 (Santa Cruz Biotechnology) antibodies and visualized with AlexaFluor488 goat anti-rabbit or goat anti-mouse AlexaFluor488 secondary antibodies (Invitrogen), respectively. Cells were visualized with an ApoTome fluorescence microscope (Zeiss). Photographs were taken by using a digital CCD camera and images were processed using the AxioVision 4.8 software. The mean corrected total cell fluorescence (CTCF) was calculated on at least 100 cells per sample, using the following equation: $\mathrm{CTCF}=$ Integrated Density of selected cell (Area of selected cell x Mean fluorescence of background readings). All measurements were performed using the ImageJ software.

\section{Flow cytometric (FACS) analysis}

Cells cultured as monolayers and their derived tumorspheres were incubated for different time intervals in the presence or absence of APO-Curcumin, free Curcumin, at a final Curcumin concentration of $50 \mu \mathrm{M}$, or Apoferritin $(0.22 \mu \mathrm{M})$. Cells were then harvested and subsequently disaggregated using enzymatic and mechanical dissociation and washed in PBS supplemented with $0.2 \%$ BSA and $0.01 \%$ sodium azide (SigmaAldrich). Cells were then stained with the Annexin V apoptosis detection kit (eBioscience) according to the manufacturer's instructions. The amount of cell death induced by the treatments was calculated as ratio among the percentage of Annexin $\mathrm{V}^{+}$and propidium iodide $(\mathrm{PI})^{+}$ cells in treated samples compared to control cells.

To quantify APO-FITC uptake, cells were cultured with APO-FITC $(1 \mu \mathrm{M}$ in FITC and $0.3 \mu \mathrm{M}$ in APO) for 24 hours, then dissociated and stained with Alexa-Fluor647conjugated anti-Sca-1 monoclonal antibody (Biolegend) as previously described [8]. All samples were analyzed using a CyAn ADP Flow Cytometer and the Summit 4.3 software (Beckman Coulter).

\section{CSC self-renewal assay}

Tumorspheres generated from MDA-MB-231 and TUBO cells were dissociated after 5 days of culture and plated at the density of $6 \times 10^{4}$ cells $/ \mathrm{mL}$ in ultra low attachment six-well dishes in presence or not of APO-Curcumin, free Curcumin, at a final Curcumin concentration of $50 \mu \mathrm{M}$, or Apoferritin $0.22 \mu \mathrm{M}$. The total number of tumorspheres in each well was counted after 96 hours of culture and reported as number of tumorspheres generated per $10^{3}$ cells plated [8].

\section{Cell transfection}

200 pmol of siRNA specific for SCARA5 (MSS291462) or negative control scrambled siRNA (Stealth RNAi Negative Control Hi-GC) were incubated with $10 \mu \mathrm{L}$ Lipofectamin 2000 and diluted in OptiMEM Reduced Serum Media (all from Thermo Fisher Scientific) according to the manifacturer's instructions. $1 \times 10^{6}$ TUBO tumorsphere-derived cells suspended in 2 $\mathrm{mL}$ tumorsphere growth medium were incubated with the transfection mix and harvested after 24,48 or 72 hours and used for further experiments.

\section{Western blotting}

Cells were lysed with cell lysis buffer (0,5\% NP40, $150 \mathrm{mM} \mathrm{NaCl}, 50 \mathrm{mM}$ Tris-HCl, 0,25 mM EDTA, 1 mM DTT, $1 \mathrm{mM} \mathrm{Na}_{3} \mathrm{VO}_{4}$ and 1:2000 protease inhibitor cocktail (all from Sigma-Aldrich)) as in [46]. $40 \mu \mathrm{g}$ total proteins were resolved by SDS-PAGE and electroblotted onto PVDF membranes. After blocking with nonfat dry milk diluted in Tris-Buffered Saline - Tween (TTBS) 0,05\%, membranes were probed with rabbit anti-SCARA5 (Santa Cruz Biotechnology) or mouse anti-vinculin (Santa Cruz Biotechnology) antibodies followed by HRP-conjugated anti-rabbit IgG or antimouse IgG (all from Sigma-Aldrich) and visualized with the ECL Western blotting substrate (Thermo Fisher Scientific), using a ChemiDoc Touch Imaging System (Biorad).

\section{In vivo experiments}

BALB/c mice (Charles River Laboratories) were maintained at the Molecular Biotechnology Center, University of Turin, and treated in accordance with University Ethical Committee and European guidelines under Directive 2010/63. Mice were subcutaneously (s.c.) challenged with $1 \times 10^{4}$ tumorsphere-derived cells (TUBO) and treated intravenously (i.v.) with APOCurcumin $(10 \mathrm{mg} / \mathrm{Kg}$ of Curcumin dose, corresponding to a protein dose of $53 \mathrm{mg} / \mathrm{Kg}$ ) or Apoferritin (at the same protein dose) every three days, starting when they have developed a $2 \mathrm{~mm}$ mean diameter tumor, or left untreated. Tumor growth was monitored twice a week with a caliper and reported as the mean of two perpendicular diameters. When tumors reached $10 \mathrm{~mm}$ mean diameter, mice were euthanized for ethical reasons.

\section{ACKNOWLEDGMENTS}

Prof. Paola Turano, Dr. Caterina Bernacchioni, Dr. Silvia Ciambellotti are acknowledged for the preparation and purification of H-Ferritin and Prof. Juan Carlos Cutrin for histological analysis of mouse livers. 


\section{CONFLICTS OF INTEREST}

All authors declare no conflicts of interest.

\section{GRANT SUPPORT}

This research was funded by MIUR (PRIN 2012 code 2012SK7ASN) and by the AIRC investigator Grant IG2013. This research was performed in the framework of the EU COST Action TD1004. L.C. was supported by a fellowship from Fondazione Umberto Veronesi.

\section{REFERENCES}

1. Frank NY, Schatton T, Frank MH. The therapeutic promise of the cancer stem cell concept. J Clin Invest. 2010; 120:41-50.

2. Maccalli C, De Maria R. Cancer stem cells: perspectives for therapeutic targeting. Cancer Immunol Immunother. 2015; 64:91-97.

3. La Porta CA, Zapperi S. Human breast and melanoma cancer stem cells biomarkers. Cancer Lett. 2013; 338:69-73.

4. Al-Hajj M, Wicha MS, Benito-Hernandez A, Morrison SJ, Clarke MF. Prospective identification of tumorigenic breast cancer cells. Proc Natl Acad Sci U S A. 2003; 100:3983-3988.

5. Nicolis SK. Cancer stem cells and "stemness" genes in neuro-oncology. Neurobiol Dis. 2007; 25:217-229.

6. Dontu G, Abdallah WM, Foley JM, Jackson KW, Clarke MF, Kawamura MJ, Wicha MS. In vitro propagation and transcriptional profiling of human mammary stem/ progenitor cells. Genes Dev. 2003; 17:1253-1270.

7. Charafe-Jauffret E, Ginestier C, Birnbaum D. Breast cancer stem cells: tools and models to rely on. BMC Cancer. 2009; 9:202.

8. Conti L, Lanzardo S, Arigoni M, Antonazzo R, Radaelli E, Cantarella D, Calogero RA, Cavallo F. The noninflammatory role of high mobility group box $1 /$ Tolllike receptor 2 axis in the self-renewal of mammary cancer stem cells. FASEB J. 2013; 27:4731-4744.

9. Lanzardo S, Conti L, Rooke R, Ruiu R, Accart N, Bolli E, Arigoni M, Macagno M, Barrera G, Pizzimenti S, Aurisicchio L, Calogero RA, Cavallo F. Immunotargeting of Antigen xCT Attenuates Stem-like Cell Behavior and Metastatic Progression in Breast Cancer. Cancer Res. 2016; 76:62-72.

10. Farnie G, Clarke RB, Spence K, Pinnock N, Brennan K, Anderson NG, Bundred NJ. Novel cell culture technique for primary ductal carcinoma in situ: role of Notch and epidermal growth factor receptor signaling pathways. J Natl Cancer Inst. 2007; 99:616-627.

11. Tallerico R, Todaro M, Di Franco S, Maccalli C, Garofalo C, Sottile R, Palmieri C, Tirinato L, Pangigadde PN, La Rocca R, Mandelboim O, Stassi G, Di Fabrizio E, et al.
Human NK cells selective targeting of colon cancerinitiating cells: a role for natural cytotoxicity receptors and MHC class I molecules. J Immunol. 2013; 190:2381-2390.

12. Todaro M, D'Asaro M, Caccamo N, Iovino F, Francipane MG, Meraviglia S, Orlando V, La Mendola C, Gulotta G, Salerno A, Dieli F, Stassi G. Efficient killing of human colon cancer stem cells by gammadelta $\mathrm{T}$ lymphocytes. J Immunol. 2009; 182:7287-7296.

13. Sangiolo D, Mesiano G, Gammaitoni L, Leuci V, Todorovic M, Giraudo L, Cammarata C, Dell'Aglio C, D'Ambrosio L, Pisacane A, Sarotto I, Miano S, Ferrero I, CarnevaleSchianca F, Pignochino Y, Sassi F, et al. Cytokine-induced killer cells eradicate bone and soft-tissue sarcomas. Cancer Res. 2014; 74:119-129.

14. Lui GY, Kovacevic Z, Richardson V, Merlot AM, Kalinowski DS, Richardson DR. Targeting cancer by binding iron: Dissecting cellular signaling pathways. Oncotarget. 2015; 6:18748-18779. doi: 10.18632/oncotarget.4349.

15. Raza M, Chakraborty S, Choudhury M, Ghosh PC, Nag A. Cellular iron homeostasis and therapeutic implications of iron chelators in cancer. Curr Pharm Biotechnol. 2014; 15:1125-1140

16. Schonberg DL, Miller TE, Wu Q, Flavahan WA, Das NK, Hale JS, Hubert CG, Mack SC, Jarrar AM, Karl RT, Rosager AM, Nixon AM, Tesar PJ, et al. Preferential Iron Trafficking Characterizes Glioblastoma Stem-like Cells. Cancer Cell. 2015; 28:441-455.

17. Geninatti Crich S, Cutrin JC, Lanzardo S, Conti L, Kalman FK, Szabo I, Lago NR, Iolascon A, Aime S. Mn-loaded apoferritin: a highly sensitive MRI imaging probe for the detection and characterization of hepatocarcinoma lesions in a transgenic mouse model. Contrast Media Mol Imaging. 2012; 7:281-288.

18. Finazzi D, Arosio P. Biology of ferritin in mammals: an update on iron storage, oxidative damage and neurodegeneration. Arch Toxicol. 2014; 88:1787-1802.

19. Tosha $\mathrm{T}$, Behera RK, Ng HL, Bhattasali O, Alber T, Theil EC. Ferritin protein nanocage ion channels: gating by N-terminal extensions. J Biol Chem. 2012; 287:13016-13025

20. Sun C, Yuan Y, Xu Z, Ji T, Tian Y, Wu S, Lei J, Li J, Gao $\mathrm{N}$, Nie G. Fine-tuned h-ferritin nanocage with multiple gold clusters as near-infrared kidney specific targeting nanoprobe. Bioconjug Chem. 2015; 26:193-196.

21. Fantechi E, Innocenti C, Zanardelli M, Fittipaldi M, Falvo E, Carbo M, Shullani V, Di Cesare Mannelli L, Ghelardini C, Ferretti AM, Ponti A, Sangregorio C, Ceci P. A smart platform for hyperthermia application in cancer treatment: cobalt-doped ferrite nanoparticles mineralized in human ferritin cages. ACS Nano. 2014; 8:4705-4719.

22. Cutrin JC, Crich SG, Burghelea D, Dastru W, Aime S. Curcumin/Gd loaded apoferritin: a novel "theranostic" agent to prevent hepatocellular damage in toxic induced acute hepatitis. Mol Pharm. 2013; 10:2079-2085. 
23. Gumulec J, Fojtu M, Raudenska M, Sztalmachova M, Skotakova A, Vlachova J, Skalickova S, Nejdl L, Kopel P, Knopfova L, Adam V, Kizek R, Stiborova M, Babula P, Masarik M. Modulation of induced cytotoxicity of doxorubicin by using apoferritin and liposomal cages. Int $\mathrm{J}$ Mol Sci. 2014; 15:22960-22977.

24. Liang M, Fan K, Zhou M, Duan D, Zheng J, Yang D, Feng J, Yan X. H-ferritin-nanocaged doxorubicin nanoparticles specifically target and kill tumors with a single-dose injection. Proc Natl Acad Sci U S A. 2014; 111:14900-14905.

25. Geninatti Crich S, Cadenazzi M, Lanzardo S, Conti L, Ruiu R, Alberti D, Cavallo F, Cutrin JC, Aime S. Targeting ferritin receptors for the selective delivery of imaging and therapeutic agents to breast cancer cells. Nanoscale. 2015; 7:6527-6533.

26. Gupta SC, Patchva S, Aggarwal BB. Therapeutic roles of curcumin: lessons learned from clinical trials. AAPS J. 2013; 15:195-218.

27. Anand P, Kunnumakkara AB, Newman RA, Aggarwal BB. Bioavailability of curcumin: problems and promises. Mol Pharm. 2007; 4:807-818.

28. Lanzardo S, Conti L, Rooke R, Ruiu R, Accart N, Bolli E, Arigoni M, Macagno M, Barrera G, Pizzimenti S, Aurisicchio L, Calogero RA, Cavallo F. Immunotargeting of antigen xCT attenuates stem-like cell behavior and metastatic progression in breast cancer. Cancer Res. 2016; 76:62-72.

29. Li JY, Paragas N, Ned RM, Qiu A, Viltard M, Leete T, Drexler IR, Chen X, Sanna-Cherchi S, Mohammed F, Williams D, Lin CS, Schmidt-Ott KM, Andrews NC, Barasch J. Scara5 is a ferritin receptor mediating nontransferrin iron delivery. Dev Cell. 2009; 16:35-46.

30. Gossuin Y, Muller RN, Gillis P. Relaxation induced by ferritin: a better understanding for an improved MRI iron quantification. NMR Biomed. 2004; 17:27-432.

31. Pozzi C, Di Pisa F, Bernacchioni C, Ciambellotti S, Turano $\mathrm{P}$, Mangani S. Iron binding to human heavy-chain ferritin. Acta Crystallogr D Biol Crystallogr. 2015; 71:1909-1920.

32. Ravera E, Ciambellotti S, Cerofolini L, Martelli T, Kozyreva T, Bernacchioni C, Giuntini S, Fragai M, Turano P, Luchinat C. Solid-State NMR of PEGylated Proteins. Angew Chem Int Ed Engl. 2016; 55:2446-2449.

33. Sigismund S, Confalonieri S, Ciliberto A, Polo S, Scita G, Di Fiore PP. Endocytosis and signaling: cell logistics shape the eukaryotic cell plan. Physiol Rev. 2012; 92:273-366.

34. Aime S, Frullano L, Geninatti Crich S. Compartmentalization of a gadolinium complex in the apoferritin cavity: a route to obtain high relaxivity contrast agents for magnetic resonance imaging. Angew Chem Int Ed Engl. 2002; 41:1017-1019.

35. Runge VM, Parker JR. Worldwide clinical safety assessment of gadoteridol injection: an update. Eur Radiol. 1997; 7 Suppl 5:243-245.

36. Esatbeyoglu T, Huebbe P, Ernst IM, Chin D, Wagner AE, Rimbach G. Curcumin-from molecule to biological function. Angew Chem Int Ed Engl. 2012; 51:5308-5332.

37. Ravindran J, Prasad S, Aggarwal BB. Curcumin and cancer cells: how many ways can curry kill tumor cells selectively? AAPS J. 2009; 11:495-510.

38. Kakarala M, Brenner DE, Korkaya H, Cheng C, Tazi K, Ginestier C, Liu S, Dontu G, Wicha MS. Targeting breast stem cells with the cancer preventive compounds curcumin and piperine. Breast Cancer Res Treat. 2010; 122:777-785.

39. Li Y, Zhang T. Targeting cancer stem cells by curcumin and clinical applications. Cancer Lett. 2014; 346:197-205.

40. Kesharwani P, Banerjee S, Padhye S, Sarkar FH, Iyer AK. Hyaluronic Acid Engineered Nanomicelles Loaded with 3,4-Difluorobenzylidene Curcumin for Targeted Killing of CD44+ Stem-Like Pancreatic Cancer Cells. Biomacromolecules. 2015; 16:3042-3053.

41. Syng-Ai C, Kumari AL, Khar A. Effect of curcumin on normal and tumor cells: role of glutathione and bcl-2. Mol Cancer Ther. 2004; 3:1101-1108.

42. Zebib B, Mouloungui Z, Noirot V. Stabilization of curcumin by complexation with divalent cations in glycerol/water system. Bioinorg Chem Appl. 2010:292760.

43. Charpentier MS, Whipple RA, Vitolo MI, Boggs AE, Slovic J, Thompson KN, Bhandary L, Martin SS. Curcumin targets breast cancer stem-like cells with microtentacles that persist in mammospheres and promote reattachment. Cancer Res. 2014; 74:1250-1260.

44. Cavallo F, Quaglino E, Cifaldi L, Di Carlo E, Andre A, Bernabei P, Musiani P, Forni G, Calogero RA. Interleukin 12-activated lymphocytes influence tumor genetic programs. Cancer Res. 2001; 61:3518-3523.

45. Astolfi A, Landuzzi L, Nicoletti G, De Giovanni C, Croci S, Palladini A, Ferrini S, Iezzi M, Musiani P, Cavallo F, Forni G, Nanni P, Lollini PL. Gene expression analysis of immune-mediated arrest of tumorigenesis in a transgenic mouse model of HER-2/neu-positive basal-like mammary carcinoma. Am J Pathol. 2005; 166:1205-1216.

46. Conti L, De Palma R, Rolla S, Boselli D, Rodolico G, Kaur $\mathrm{S}$, Silvennoinen O, Niccolai E, Amedei A, Ivaldi F, Clerico M, Contessa G, Uccelli A, Durelli L, Novelli F. Th17 cells in multiple sclerosis express higher levels of JAK2, which increases their surface expression of IFN-gammaR2. J Immunol. 2012; 188:1011-1018. 